

\section{Productividad Académica Departamento de Diseño UCh / 2012}

Con motivo de un proyecro Fondecyc, el profesor Clatadio Cortés adscrito al Departamento de Diseño, esturo en la Universidad de Granada realizando, junto a otros investigadores chilenos, una estadía de investigación. Esta labor tuvo cono rema cerztral el fenómeno de la crítica de arre, el cual fue analizado desde diferentes perspectivas. Al mismo tiempo discuteron el matco metodológico con especialistas españoles, como también los alcances y limites de este proyecto, el cual debe ser terminado en diciembre de $20 \%$.

\section{Publicación de nuevo libro}

El académico Cristián Gómez-Moya pertenecience al Departamento de Diseño, ha publicado el libro "Derechos ae mirada. Arte y visulidad en los archiros desclasificados". La publicación, del sello editor Palinodia, recoge el rabajo en carso del autor, que ya se ha manifestado en la curatoxka del espacio de consulca "Huran Rights Copy Rights" en el marco de la exposición "Chite años 70 y 80 . Memoria y experimentalidad" "ea lizada en el mate de la Universidad de Chile, asi como en ozzas iniciativas similares.

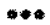

\section{Workshop en EE.UU.}

El acadénico del Departamenzo, Diego Gómez, estuvo de visita en la ciudax de Boston, Massachusetts, durarte la semana del zas al 27 de abri? realizando un worksop en conjunto con la diseffiadora y artista norteamericana Lauren
McCarthy. El taller se áenominó "Empoderamiento Tecnológico y Acrivismo a través de Visualización de Datos" (Vistal Hacktivism en inglés) y su realización se hizo en el contexto de NuVu Szudios, un centro de innovación en étuctación.

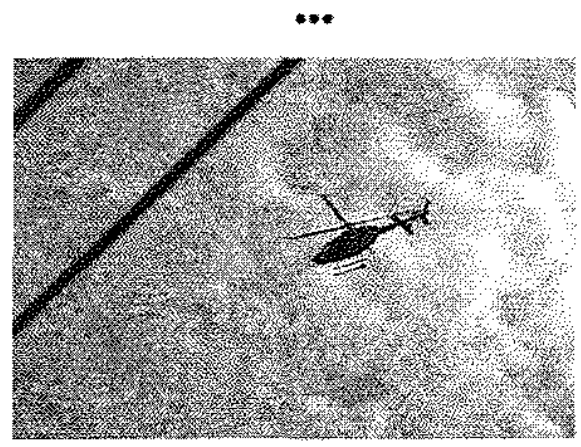

\section{Charla en España}

La ponencia ora de la académica Rebeca Silva se zealizó con motivo del segundo Congreso Interracional de Diseño e Innowación de Cataluña (CIDlC) er la ciudad de Batcelona, Espana, everro desarrollado en los dias 25,26 y 27 de abril de $20 \mathrm{n} 2$.

Esta pontencia es producto de la Inwestigación *Arsapaluz; La luz como instrumento de transformación y de apropiación del espacio conseruido para se revitulización en la experiencia del usiario. Transversalidad metodológica entre arquirectura y disefosos (Proyecto kav: Código o5/ogl y en d marco del $\mathrm{N}$ Concurso de IN. CeNTIVO AL PATENTAMIENTO UNTVRSGLAD DE CHILE - 2011, con registro en el Instiruto 
Nacional de Propiedad Industrial (rNapl), bajo

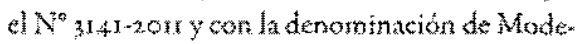
lo de Utilidad, cuya solicitud se encuentra en curso. Esta investigación y su pastentamiento han sido desarrollados en conjunto con las profesoras Sofia Letelier Parga y Cecilia Wolff Cetch de: Departamento de Arquiftectura.

\section{Dos FONDART para académico del DDD}

Eduardo Castillo, profesor del Departamento de Diseño, es responsable die dos proyectos seleccionados por los Fondos de Cultura (FONDART) para ser desarrollados durante cste ano. Los proyectos ganadores son "Francisco Otta, Obra Gráfica" " "Taller Editorial Matricio Amster" este ultimo dentro del Fondo Nacional de Fomerto del ibro y la lectura, por un tozal combinado de casi 20 mitlones de pesos.

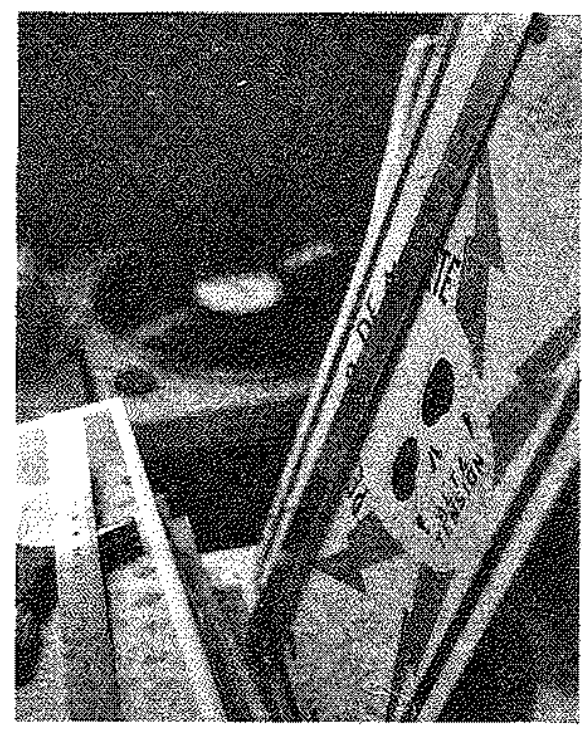

\section{Proyectos Ganadores Fondos VID}

El proyecto HUMAN RIGHTS / CONY RIGHTS. ARCHNOS VISTAZES EN LA EPOCA DELADESCEASIFICACrón se ha adjudicado al Concurso de proyecros de Creación vio zon, que anual mente entrega la Vicerrectoria de Invastigación y Desanolo de la Universidad de Chute. Bajola responsabilidad de Cristín Gómez-Moya, academico biel $\mathrm{bDD}$, esre proyecso de creación contempla una investigación editorial sobre archivos visuales, cuyo principal foco es la desclasificacion posticutatoulal de los informes secretos derivados del Nutional Security Archizte (Us 1999-2010).

A través del ejercicio de traducción y homo grafia que enuncia entre derechos humanos y derechos de eppias, el proyecro ofrecerá un do cumento de archivo sobre las políticas de la imagen y la visualidad, lo cual persigue desnatz. ralizar la menonia histórica de estos documentos biopoliticos y su circulación visiza en los dominios globales.

E proyecto es parte de la línea de desarollo que el asademico ha generado a traves de la plata. forma Archivo de Iavestigaciones Visuales (AIV), que ha comprendido la proyección cutatorial en dMusto de Artc Contemporánco (Mac) durante el zoxk, asi como una futura serie de intervenciones tarto en Chile como en Estados Unidos a partir del segundo semesre del zor:.

Por otra parte, los académicos Alejandro Pantoja y Dicgo Gómez tambicn han resultado ganadores de un Fondo de Creación viD. Et profesor Pantoju es el creador responsable, mientras que el profesor Gómez participaraz como cocreador.

El proyecto presenado puede definirse como una Vesualización de datos con carácter de civis- 
mo medial. El nombre de trabajo del proyecto es: "Visualización de Daros, Semáncica y Medios de Prensa Online Chilenos". La idea detrás de esta iniciativa es desarrollar un sistema de análisis del contenido noticioso escrito, de modo tal que sea posible encontrar patrones semánticos que serán luego traspasados a un sistema visual que permita visualizar las "zonas discursivas" donde se mueven estos medios, en relación con su contenido. Para programar se usará Processing, usando lenguaje natural y Regular Expressions. Con esto, se construirá un sitio web en HTML. El proyecto se desarrollará desde marzo hasta occubre del 2012 y contará con la ayuda de dos estudiantes de la carrera de diseño. Finalmente, cabe destacar que este es el primer proyecto desarrollado por el People and Media Lab.

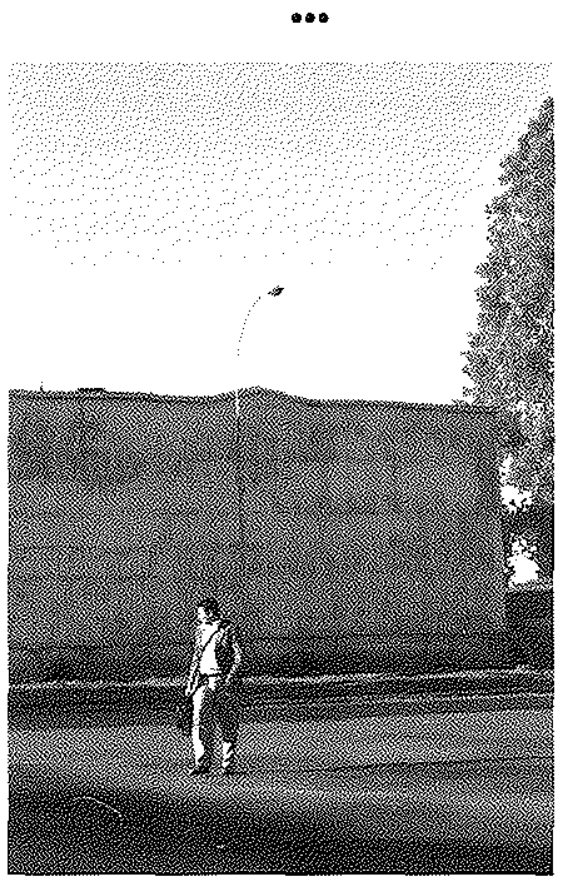

\section{Conferencia en Congreso Anual de la} Sociedad de Historia del Diseño

El profesor Mauricio Vico, académico del Departamento de Diseño de la Fau, llevó a cabo una conferencia titulada: "El cartel político chileno bajo el gobierno de Salvador Allende (x970-1973)" en el Congreso anual de la Sociedad de Historia del Diseño, el que tuvo como tema "Diseño activista y Cambio social". Este evento, que por primera vez se celebra fuera de la ciudad de Londres, se realizó en la Universidad de Barcelona, Facultad de Historia y Geografía, entre el 7 y ro de sepriembre del 2011 y fue organizado por la Fundación Historia del Diseño.

A continuación presentamos un resumen de la temática presentada por el Prof. Vico en la conferencia:

"Con la elección de Salvador Allende en sepriembre de 1970 representando al conglomerado político de la Unidad Popular (UP) que aglutinó gran parte de los partidos de izquierda chilenos, le cupo al cartel la importante función de comunicar ese proyecto. Este fue un lenguaje claro y preciso, teniendo en consideración la recepción de aquellos mensajes; la gente postergada, el obrero, el campesino, la mujer pobladora, los jóvenes. El cartel fue uno de los medios más comprometidos con el gobierno y eco de las demandas sociales.

El componente político de sus carteles hizo que sus propuestas vistrales durante el período del gobierno de la UP, entre los años $x 970$ y 1973 , terminado abruptamente en un golpe de Estado, experimentaxán cambios profundos en su enfoque, en sus formas de composición, constituyendo un nuevo discurso de la visualidad como nunca hasta entonces había tenido la gráfica chilena. Lo confrontacional (oposiciones formales y de contenido), lo identizario (generación de fuertes 
relaciones de identidad), lo corporativo (medio para la difusion dol programa de la vp), lo innovador (estrategia comunicacional, gráfica inćdita) y un edlecticismo expresado en la sintesis formal de ctilos y tendencias, son aigunos de los clementos nás sobresalientece

Al carrel se incorporaron diversas tendencias: el "Arre Pop", la tradición vernácula como un signo de identidad cuitural, la Psicodelia, el mensaje político influenciado por el Cartel $\mathrm{Cu}$ bano. En su exprestón tipográticallega de Fstilo Tipográfico lnvernacional a través del catálogo de Letraset y revistas internacionales de Diseño.

Li nuevo enfoque turo el sertido de vincular dirctamente la visualidad a la contirgencia polírica, como un tenovado medio de expresión y de comunicación. Los mensajes de estos carteles apelaron a la razón y a la emotividad de sus recepcores. En sllos subyace el inismo discurso ideológico: el kompromiso con el programa socialista del gobierno".

La publicación ATLAS OF TRANSFORMATION constituye un vasto proyecto editorial organizado por TRANZIT, CZ, un ketzugrk de trabajo independiente (htep:/ www.ranzit.org) que desde el 2002 se ha desarrollado entre los paises de Auscria, Hurn gria y Republica Checa. La publicación ha consistido on la clasificación de keyworta tanto de los procesos económicos y geopolíicos así cono de los sentidos parrimoniales, culturales $\mathrm{e}$ ideológicos, de aqueellos paises que durante ultimas décadas han sido denominados "countries of transformation".

Como herramienta intclectual esta publica. ción ha perseguido rastrear procesos de cambios sociales y polizicos desde la perspectiva de los nuevos mapas transnacionales y las distintas

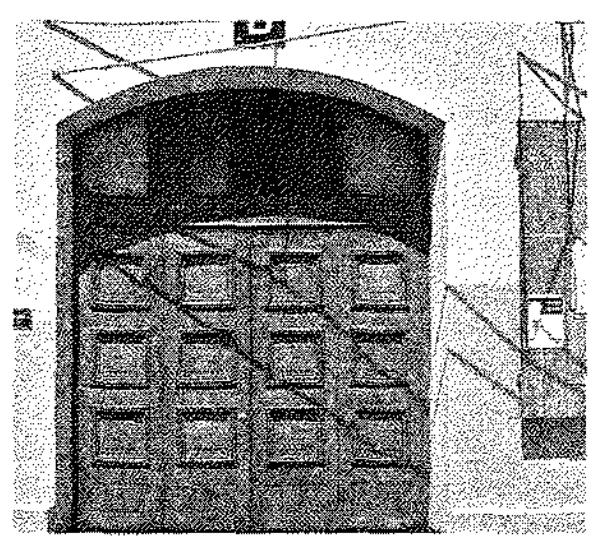

subjetividades que alli se manifiestan. Comprendisndo una amplia lista de investigadores y artistas, así como de renombrados pensadores y teóricos de nivel mundial, quienes tán contribuido a repensar estos procesos por medio de diversos textos, archivos, documentos e imágents, este libro se distancia de los formatos cariónicos de las enciclopedias modernas y plantea, en cam. bio, el díscño de una contra-cartografia de las procesos hegemónicos transnacionales.

Detos editoriales:

Athes of Trangformation

Plans for publisbing: tratzitice.

Catech edition, Praydu 20 of

Ienglish edition, 20.7o]

Distribution:JRP-Ringier

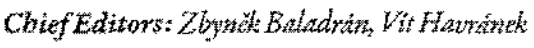

Project Direction: Vera Kriticout

Grapbic designt Adela Szobodowa

Printer: Hellych, ats, satr

En esta pablicacion ha sido invitado a participar el acadérnico \& investigador Cristián Gómez-Moya (Dod, FAC, Universidad de Chile), quier ha contribuido con alganas 
palabras ciaves asociadas a las poincicas de la inagen y la destrucción. Esta invización no solo es relevante por la tryergadura de los autores inter. racionales inciudos en a publicacion, sino además por el dispositivo intelecrual gue contempla un diseño editorial de este tipo, cuyo formato ha contribuido a pensar la transformación de un ámbito del saber como es at alas enciclopédico, care las inágenes y los imaginarios que de f́l se desprenden.

A continuacion se reproduce un breve fragmento de la entrada correspondiente a "ruinas".

\section{RUINS}

\section{Cristian Gómez-Moya}

Durante la tendición moderna curocentrada, 站

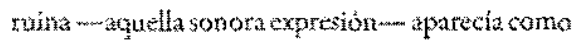
un vestigio representative de una obraperdidaen la ausencia, no obstante si imagen ha wento circulado, no sin poca nostalgia pretérita, cono el cadáwerencido de una f́pocas senalada; en ela subyace un vestigio ideologico antes que un vestigio arquitectónico. Esee deseo de ver en et pasado ciene su mirada sobre lo auténtico, lo primignio, aquello supuestamente libre de constriction policica y enmarcado, a la vez, cn tina tzaza monumenta en que la mirada hacia actis operála como una paradoja enigmárica, en tanto que nostakgingor la ausercia de un riempo incom pleto de lo moderno. Siguiendo al tebriko alemán Andreas Fuysser, "el presente imaginado de un pastou que hoy sólo puede caparse en su descomposición. Por eso la ruina es un objeto de nostalgita" (2007), empero esta mimma mirada hacia el pasado conseguiria su vigencia apelando ato aurentico como lo nuevo cauc está por venir (desde un pasado fosilizado).

Asi, si acepramos el dictum de estas diders pre- liminares - que no es otra que el referido a la imagen dialéctica entre progresoncatástrofe de ha exis benjaminiana-.., la ruina perreneceria al do. mirtio de las costa desde su dimenstón históricita es decir en aparicncia bastaría simplemeneson seguir log vestigios de la atgutrectura, el arre la tecnologia, por ejemplo, y uescubrir en ello ba historia de un pasado. La ur-historia, catezorata dave para referirse a los objecos industriales como "la huella de una historia vivience que puede ser ledia desde la superficiede los objetos sobrevivien. tes" (Benjamin, 193,), sin embargo sellaria sa pasado no sin una fuerce carga de ideologíg. Esa ruina, por unto, surgida en el espacio de ransiro. ricdad de una coyuntura politita que va del cutaber de la historia a la tecuperación del vestigto (renodelación, regentwacion, preservación y actualizacion como incorporación novedosa para la visualizacion de a hastoria, indica entonces que su condicion se encuencra deseminada por un devenir que -en el sentido ruinificado, unversalista y hegemónico que cstanos examinatido --ińa des una naruralega en estado perrificndo a wa na turaleza tro pernanente transitoredad. Animismo,

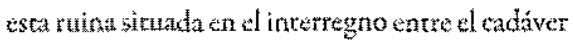
(calavata) y la novedad, eicrerería como vestigio comecror y transformador.

Para una revision completa del rabayo fversion en ingles) wéase es link:

btphifmonumenttomansformation.orghatas-of matasformation 